\title{
Melatonin supplementation modifies experimental chronic colitis in mice
}

\author{
M. Golab, M. Dudziak and K. Skwarlo-Sonta \\ Department of Animal Physiology, Faculty of Biology, University of Warsaw, Miecznikowa 1, 02-096 Warsaw, Poland
}

Melatonin (Mel) has been adopted by most living organisms as a circadian timing signal, but is also known to be a potent free radical scavenger and immunomodulatory agent ${ }^{(1)}$. Mel is not only synthesized in the pineal gland, but also in the gastrointestinal tract (GIT), where it does not depend on the light-dark cycle and may be induced by food intake or withdrawal. GIT-derived Mel may also have paracrine and autocrine functions, modifying the intestinal blood flow or mucosal regeneration ${ }^{(2)}$. The potent free radical scavenging activity of Mel may give rise to its protective and therapeutic application in chronic inflammation of the GIT ${ }^{(3)}$.

The aim of the present study was to examine the immunomodulatory properties of Mel when administered differently in experimental colitis in mice. Experiments were conducted on 6-8-week-old male BALB/c mice (five to six individuals per group) housed in standard laboratory conditions $\left(22^{\circ} \mathrm{C}, 12 \mathrm{~h}\right.$ light $-12 \mathrm{~h}$ dark). Chronic colitis was induced by administration of dextran sodium sulfate (DSS; $0.06 \mathrm{~g}$ per mouse per $\mathrm{d}$ ) in the drinking water for 2 or 3 weeks. From the beginning of the experiment some groups of mice were simultaneously (preventively) treated with Mel $(10 \mathrm{mg} / \mathrm{kg}$ body weight) by oral administration (Expt 1) or in the drinking water during the night (Expt 2). Another group of mice received Mel therapeutically after showing the first clinical symptoms of colitis (positive occult blood tests, rectal bleeding). Mice were killed by $\mathrm{CO}_{2}$ asphyxia, colons were isolated and proximal and distal fragments used for histological tests. Additionally, spleens were isolated and splenocytes cultured in the presence of mitogens. Colon mucosa was gently scraped into a protease-inhibitor solution, centrifuged and supernatant fractions used for the measurement of cytokine concentrations using flow cytometry with a mouse T-helper (Th1)/Th2 cytokine cytometric bead array kit (Becton Dickinson, Franklin Lakes, NJ, USA).

In control mice (without colitis) Mel caused immune cell proliferation in gut lymphatic nodules without any change in cytokine concentration and crypt structure. DSS administration resulted in an elevation in mucosal TNF $\alpha$, a decrease in IL-2, subsequent shortening and crypt loss as well as intensive mononuclear infiltration to the mucosa. Preventive Mel supplementation caused no changes in TNF $\alpha$ concentration in DSS-treated mice, but completely reversed the DSS-induced decrease in mucosal IL-2 content. Mel did not prevent inflammatory infiltration, but crypt loss was located either in the proximal or distal part of the colon, depending on the period of Mel treatment. Therapeutic Mel administration did not change IL-2 concentration but caused a significant decrease in TNF $\alpha$ and interferon- $\gamma$ concentrations. No significant changes in Th2-specific cytokines (IL-4, IL-5) after preventive or therapeutic administration of Mel were observed. In Expt 1 possible Mel-induced systemic changes in immunity were perturbed by the stress associated with oral administration. Splenocyte proliferation was changed by colitis and Mel in Expt 2, as a result of the less-invasive method of Mel treatment. Both B- and T-cell proliferation were decreased by Mel in mice without colitis. Splenocyte proliferation was elevated in the DSS-treated animals and decreased by Mel only if administered preventively.

These results show a possible protective action of Mel in the GIT, but its effects are dependent on the method and period of administration. Further experiments are in progress.

Supported by MNiN grant 2P04C 11729 and by Faculty of Biology, University of Warsaw intramural grant BW \#1720/20 to M.G.

1. Altun A, Ugur-Altun B (2007) Int J Clin Pract 61, 835-845.

2. Bubenik GA (2002) Dig Dis Sci 47, 2336-2348.

3. Nosál'ová V, Zeman M, Cerná S, Navarová J, Zakálová (2007) J Pineal Res 42, 364-370. 